\title{
PREVALÊNCIA DA SÍFILIS GESTACIONAL E CONGÊNITA NA POPULAÇÃO DO MUNICÍPIO DE MARINGÁ - PR
}

\section{Michelli Gouveia Ramos}

Discente do curso de Biomedicina no Centro Universitário de Maringá (UniCesumar), Brasil.

\section{Sara Macente Boni}

Docente do Departamento Biomedicina no Centro Universitário de Maringá (UniCesumar), Brasil.
RESUMO: O objetivo deste estudo foi relatar a prevalência de sífilis gestacional e congênita dos anos 2013 a 2016, com base em dados do setor de epidemiologia do município de Maringá - PR. Foi realizado um estudo descritivo retrospectivo por meio de coleta de dados em fichas de notificação compulsória de sífilis gestacional e congênita, onde foram coletados dados da pesquisa. Foram notificados 226 casos de sífilis em gestantes, cuja média de idade mais acometida é de 25,6 anos, mostrando uma prevalência elevada de sífilis primária (68,6\%), quando comparado às demais fases da doença. A sífilis congênita apresentou aumento crescente entre os anos estudados, sendo notificados 134 casos da doença em recém-nascidos. Dentre esses, 8,9\% apresentaram manifestações clínicas e alterações ósseas importantes. Com esse estudo, foi possível observar aumento significativo de sífilis gestacional e congênita no decorrer dos anos estudados, o que indica alguns pontos frágeis na assistência pré-natal e saúde da gestante, que necessitam de melhoras.

PALAVRAS-CHAVE: Gestantes; Infecções sexualmente transmissíveis; Sífilis; Treponema pallidum.

\section{PREVALENCE OF PREGNANCY AND CONGENITAL SY- PHILIS IN THE POPULATION OF MARINGÁ, BRAZIL}

\begin{abstract}
The prevalence of pregnancy and congenital syphilis between 2013 and 2016 are analyzed, foregrounded on data retrieved from the epidemiological sector of the municipality of Maringá PR Brazil. A retrospective descriptive study was undertaken by data collection from compulsory notification charts on pregnancy and congenital syphilis. Two hundred and twenty-six syphilis cases were notified in pregnant women, mean age 25.6 years, with a high prevalence primary syphilis (68.6), when compared with other phases of the disease. Congenital syphilis increased during the years under analysis, with 134 cases in newly born children, among whom $8.9 \%$ had clinical manifestations and important bone alterations. Results show significant increase in pregnancy and congenital syphilis during the studied period and reveal weak points in pre-natal assistance and health. Improvements are required.
\end{abstract}

KEY WORDS: Pregnant women; Sexually transmitted diseases; Syphilis; Treponema pallidum. 


\section{INTRODUÇÃO}

A alta incidência das Infecções Sexualmente Transmissíveis (IST) têm se tornado grande problema de saúde pública ${ }^{1}$, estando entre as cinco principais causas de procura por serviço médico ${ }^{2}$. Estima-se que, por dia, no mundo, 1 milhão de pessoas infecta-se com IST, sendo as doenças mais prevalentes a sífilis, gonorreia, tricomoníase e clamídia ${ }^{3}$. Estas enfermidades podem provocar sérias complicações, tais como infertilidade, aborto espontâneo, malformações congênitas e até a morte se não tratadas ${ }^{4}$, além de elevar o risco de contrair e transmitir o Vírus da Imunodeficiência Humana (HIV) ${ }^{5}$.

A sífilis ou lues é uma doença infecciosa sistêmica, de evolução crônica, que ocupa uma importância significativa entre os problemas mais frequentes de saúde pública em todo o mundo ${ }^{6}$. Seu agente etiológico é Treponema pallidum, uma espiroqueta adquirida durante relações sexuais desprotegidas ${ }^{\top}$ ou por transmissão vertical $^{8}$, pela disseminação hematogênica da bactéria na circulação sanguínea da mãe que atravessa a barreira placentária infectando o feto 9 podendo acontecer em qualquer momento da gravidez ${ }^{10}$. A transmissão também pode ocorrer pelo aleitamento materno, caso a parturiente apresente lesões mamárias ${ }^{11}$.

Dependendo do tempo de evolução da doença, a sífilis pode ser classificada em primária, secundária, latente e terciária, apresentando diferenças nas manifestações clínicas e imunopatológicas ${ }^{12-13}$. A sífilis primária se caracteriza pelo surgimento do cancro duro, sendo a lesão mais específica dessa enfermidade, que pode evoluir para sífilis secundária caso não seja tratada ${ }^{13}$. As manifestações na fase secundária da doença aparecem de quatro a dez dias após o surgimento do cancro primário, pela disseminação da bactéria no sistema linfático e sanguíneo ${ }^{12}$, podendo evoluir para síflis latente, com ausência de manifestações clínicassintomáticas ${ }^{14}$. Já na síflis terciária, a infecção acomete pele e mucosas podendo provocar lesões no sistema nervoso (neurossífilis) e cardiovascular, ocorrendo nessa fase, a formação do granuloma destrutivo ${ }^{13}$.

A síflis congênita é decorrente da infecção materna pela bactéria durante a gestação ${ }^{15}$ causando sérios problemas para o feto, como o aumento da morbidade na vida intrauterina que contribuem para o aborto, nati e neomortalidade, e complicações nos nascidos vivos ${ }^{16-17}$, dentre elas, partos prematuros, baixo peso, lesões cutâneo-mucosas, problemas respiratórios, anemia, hepatoesplenomegalia, deformidades ósseas, além de elevar os níveis de morte dos recém-nascidos ${ }^{9}$, o que fez com que a sífilis gestacional se tornasse uma doença de notificação compulsória conforme previsto na portaria MS/NSV $\mathrm{n}^{\circ} 33$, assinada em julho de $2005^{18}$.

A Organização Mundial da Saúde (OMS) estima que ocorra aproximadamente 1 milhão de infecções por sífilis entre as gestantes, sendo mais atingidas mulheres com níveis socioeconômicos reduzidos e estilos de vida que contribuem para a infecção, tais como múltiplos parceiros, resistência ao uso de preservativos e promiscuidade masculina ${ }^{8,19,20}$, além de apresentar antecedentes obstétricos de risco, início tardio do acompanhamento pré-natal, redução do número de consultas, tratamento inadequado dos casos diagnosticados e o não tratamento dos parceiros $^{15,21,22}$.

A incidência dos casos de sífilis congênita no Brasil é crescente, sendo notificados entre os anos de 2005 a junho de 2017, 200.253 casos de síflis em gestantes e entre 1998 e junho de 2017, 159.890 casos de síflis congênita em menores de um ano de idade. A elevação da taxa de incidência de sífilis congênita e as taxas de detecção de sífilis em gestante por mil nascidos vivos aumentaram cerca de três vezes entre os anos de 2010 e 2016, passando de 2,4 para 6,8 e de 3,5 para 12,4 casos por mil nascidos vivos, respectivamente ${ }^{23}$, caracterizando a sífilis como uma doença re-emergente, necessitando de rastreamento e tratamento em tempo hábil a fim de conter a infecção $0^{7,24}$.

Segundo o Ministério da Saúde, toda gestante deve ser submetida ao menos a dois testes Venereal $D i$ sease Research Laboratory (VDRL) durante a gestação, devendo ser realizado um no primeiro trimestre e outro no terceiro trimestre da gravidez, além da realização do exame antes do parto para garantir que o recém-nascido seja tratado precocemente caso a gestante não tenha recebido tratamento para sífilis na gestação ${ }^{25}$.

O diagnóstico da sífilis congênita pode ser feito por meio da pesquisa direta das espiroquetas em microscopia de campo escuro de amostra proveniente da pla- 
centa e cordão umbilical e por VDRL, que consiste em um teste não treponêmico. O teste de absorção de anticorpos treponêmico fluorescente (FTA-Abs) não é realizado em recém-nascidos pelo risco de resultados falsos negativos $^{11,26}$. Considera-se sífilis congênita confirmada a evidência de espiroquetas em amostras de tecidos, líquidos ou fluídos corporais ${ }^{15}$.

Apesar do diagnóstico e tratamento ser conhecido e a doença ser de fácil prevenção $0^{27}$, seu controle ainda é um grande desafio para a vigilância epidemiológica e serviços de saúde ${ }^{28}$, o que se justifica por deficiências na assistência pré-natal ${ }^{8,29}$, visto que é uma doença que pode ser evitada desde que a gestante seja diagnosticada e tratada antes do parto ${ }^{3,30}$.

$\mathrm{O}$ presente estudo teve como objetivo relatar a prevalência de sífilis gestacional e congênita, com base em dados do setor de epidemiologia do município dos anos 2013 a 2016, relacionando-as com os casos registrados no Brasil, com o intuito de salientar a importância da procura pelo diagnóstico e tratamento precoce para reduzir a transmissão da doença.

\section{METODOLOGIA}

Foi realizado um estudo descritivo e retrospectivo das notificações compulsórias de síflis gestacional e congênita durante os anos de 2013 a 2016, na cidade de Maringá, Estado do Paraná (PR). Foram incluídas todas as notificações realizadas neste período não sendo delimitada a idade dos pacientes e excluídos da pesquisa notificações de moradores dos distritos pertencentes ao município.

A coleta foi realizada por meio das fichas de notificações da enfermidade estudada do setor de epidemiologia do município de Maringá após a aprovação do comitê de ética (parecer $\left.n^{0} 2.067 .629\right)$. Os dados coletados foram mês e ano das notificações, idade das gestantes, escolaridade, etnia, resultados dos exames sorológicos das gestantes e dos recém-nascidos, esquema de tratamento da gestante e do parceiro, caso não tenha sido tratado concomitantemente à gestante, qual o motivo para o não tratamento, sexo dos recém-nascidos, resultados dos exames do sangue periférico e líquor, resultado do exame radiológico de ossos longos e principais manifestações clínicas.
Os dados foram descritos como frequência absoluta e percentual em relação à amostra total.

\section{RESULTADOS}

Foram analisadas fichas de notificação compulsória de 226 casos de sífilis em gestantes e 134 de sífilis congênita do setor de epidemiologia do município de Maringá - PR, no período de janeiro de 2013 a dezembro de 2016.

As gestantes acometidas com a doença estudada apresentaram idade média de 25,6 anos. Em todos os anos estudados houve maior prevalência da enfermidade em gestantes de 20 a 30 anos (Tabela 1). Quando analisada a classificação clínica da doença, a sífilis primária apresentou prevalência de 68,6\% (155/226), enquanto a sífilis secundária de 7,1\% (16/226), sífilis terciária de 4\% (9/226), 5,3\% (12/226) encontravam-se na fase latente da enfermidade e em 15\% (34/226) das notificações esta informação foi ignorada.

Tabela1. Faixa etária das 226 mulheres acometidas por síflis durante a gestação na cidade de Maringá - PR, no período de 2013 a 2016

\begin{tabular}{|c|c|c|c|c|c|c|c|c|}
\hline \multirow{2}{*}{$\begin{array}{c}\text { Caracterís- } \\
\text { ticas }\end{array}$} & \multicolumn{2}{|c|}{2013} & \multicolumn{2}{|c|}{2014} & \multicolumn{2}{|c|}{2015} & \multicolumn{2}{|c|}{2016} \\
\hline & $\mathbf{n}$ & $\%$ & $\mathbf{n}$ & $\%$ & $\mathbf{n}$ & $\%$ & $\mathbf{n}$ & $\%$ \\
\hline \multicolumn{9}{|c|}{ Faixa etária (anos) } \\
\hline Até 19 & 5 & 13,5 & 11 & 23,4 & 8 & 13,1 & 12 & 14,8 \\
\hline $20-30$ & 22 & 59,5 & 23 & 48,9 & 42 & 68,9 & 53 & 65,4 \\
\hline$>30$ & 10 & 27,0 & 13 & 27,7 & 11 & 18,0 & 16 & 19,8 \\
\hline
\end{tabular}

Fonte: Dados da pesquisa

Muitos parceiros das mulheres estudadas não receberam tratamento concomitantemente à gestante (69,5\%) favorecendo a reinfecção da mesma. O motivo para o não tratamento, na maioria dos casos, foi por não possuir mais contato com a gestante ou o não comparecimento destes na unidade básica de saúde para a realização da terapia adequada. Este critério constava como ignorado em 3\% das notificações analisadas.

Em relação aos exames laboratoriais realizados durante a gestação, o VDRL apresentou títulos superiores a 1/8 atingindo titulação de 1/2560. Das gestantes 
que realizaram este teste durante o pré-natal, $77,4 \%$ (175/226) obtiveram resultado reagente, enquanto $22,6 \%$ (51/226) foram não reagentes. No exame confirmatório para a doença (FTA-Abs), 77,6\% (180/226) das gestantes tiveram o teste reagente, $12 \%$ (27/226) não reagente e 8,4\% (19/226) não realizaram o exame. Quando comparado o resultado dos dois exames, em 69,9\% (158/226) das fichas analisadas foram compatíveis, sendo ambos os testes positivos em 91,1\% e negativos em 8,9\%. Das 68 gestantes com resultados de VDRL e FTA-Abs discrepantes, 52,9\% apresentaram VDRL não reagente e FTA-Abs reagente, 26,5\% VDRL reagente e o FTA-Abs não foi realizado, 19,1\% VDRL reagente e FTA-Abs não reagente 1,5\% apresentaram negatividade no VDRL e não foi realizado FTA-Abs.

Os dados presentes nas fichas de notificação do pré-natal das gestantes dessas mulheres foram precários, pois apesar de demonstrarem que 81,9\% (185/226) das gestantes diagnosticadas com síflis foram tratadas, faltavam informações do início e número de consultas, não havia relatos sobre o período de gestação e não havia informação clara sobre a continuidade do tratamento materno depois da positividade para os exames. Além disso, houve a presença constante de dados ignorados ou em branco.

Das 134 notificações de sífilis congênita durante o período do estudo, identificaram-se tratar de 54,5\% bebês do sexo masculino e 45,5\% do sexo feminino. Em sua totalidade, $131(97,8 \%)$ gestantes fizeram acompanhamento pré-natal, três mulheres tiveram este dado ignorado em sua ficha de notificação da doença, sendo todas estas notificações realizadas em 2015.

As vaiáveis relacionadas às características maternas coletadas de fichas de notificações de síflis congênita evidenciaram que houve predomínio da escolaridade ensino fundamental incompleto (27,6\%), faixa etária entre 20 a 30 anos $(64,2 \%)$ e parturientes de etnia branca (56\%) (Tabela 2).

Tabela 2. Características epidemiológicas maternas das 134 notificações de sífilis congênita na cidade de Maringá - PR, no período de 2013 a 2016

\begin{tabular}{|c|c|c|c|c|c|c|c|c|}
\hline \multirow{2}{*}{ Características } & \multicolumn{2}{|c|}{2013} & \multicolumn{2}{|c|}{2014} & \multicolumn{2}{|c|}{2015} & \multicolumn{2}{|c|}{2016} \\
\hline & n & $\%$ & n & $\%$ & $\mathbf{n}$ & $\%$ & $\mathbf{n}$ & $\%$ \\
\hline \multicolumn{9}{|c|}{ Faixa etária (anos) } \\
\hline Até 19 & 1 & 9,1 & 2 & 13,3 & 8 & 15,4 & 7 & 12,5 \\
\hline $20-30$ & 6 & 54,5 & 9 & 60,0 & 34 & 65,4 & 37 & 66,1 \\
\hline$>30$ & 4 & 36,4 & 4 & 26,7 & 10 & 19,2 & 12 & 21,4 \\
\hline \multicolumn{9}{|l|}{ Escolaridade } \\
\hline EFI & 4 & 36,4 & 3 & 20,0 & 11 & 21,2 & 19 & 33,9 \\
\hline EFC & 2 & 18,2 & 3 & 20,0 & 11 & 21,2 & 7 & 12,5 \\
\hline EMI & 1 & 9,1 & 3 & 20,0 & 14 & 26,9 & 12 & 21,4 \\
\hline EMC & 3 & 27,2 & 1 & 6,7 & 14 & 26,9 & 14 & 25,0 \\
\hline ESI & 0 & 0,0 & 0 & 0,0 & 0 & 0,0 & 1 & 1,8 \\
\hline ESC & 0 & 0,0 & 1 & 6,7 & 1 & 1,9 & 3 & 5,4 \\
\hline Ignorado & 1 & 9,1 & 4 & 26,6 & 1 & 1,9 & 0 & 0,0 \\
\hline \multicolumn{9}{|l|}{ Cor/Raça } \\
\hline Branca & 6 & 54,5 & 10 & 66,7 & 27 & 51,9 & 32 & 57,1 \\
\hline Parda & 5 & 45,5 & 3 & 20,0 & 20 & 38,5 & 23 & 41,1 \\
\hline Amarela & 0 & 0,0 & 0 & 0,0 & 1 & 1,9 & 0 & 0,0 \\
\hline Negra & 0 & 0,0 & 2 & 13,3 & 4 & 7,7 & 1 & 1,8 \\
\hline
\end{tabular}

EFI: Ensino Fundamental Incompleto; EFC: Ensino Fundamental Completo; EMI: Ensino Médio Incompleto; EMC: Ensino Médio Completo; ESI: Ensino Superior Incompleto; ESC: Ensino Superior Completo. 
As notificações de sífilis congênita apresentaram aumento crescente no número de casos, sendo notificados 11 casos em 2013, 15 em 2014, 52 em 2015 e 56 casos em 2016 (Figura 1). De todos os casos, sete apresentaram alterações ósseas importantes e cinco tinham manifestações clínicas no momento do diagnóstico, dois bebês apresentavam icterícia, um tinha lesões cutâneas, um a função hepática alterada e um tinha alterações musculares.

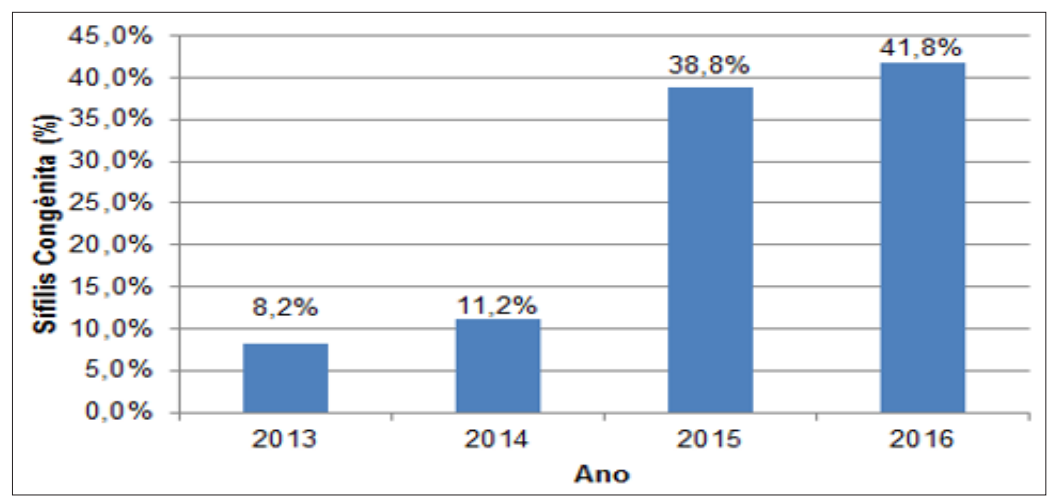

Figura 1. Prevalência de sífilis congênita na população de Maringá-PR, no período de 2013 a 2016.

O teste confirmatório de sífilis congênita consiste na pesquisa de espiroquetas em amostras da placenta e cordão umbilical dos recém-nascidos e em 39\% (52/134) dos casos notificados não foram realizados, 23\% (31/134) tiveram a informação ignorada, 29\% (39/134) não houve a evidência do Treponema pallidum e 9\% (12/134) foi positivo para o exame (Figura 2). Já é conhecido que a maioria dos diagnósticos de sífilis congênita é presuntivo, já que a demonstração definitiva de infecção pelo Treponema pallidum é rara ${ }^{31}$.

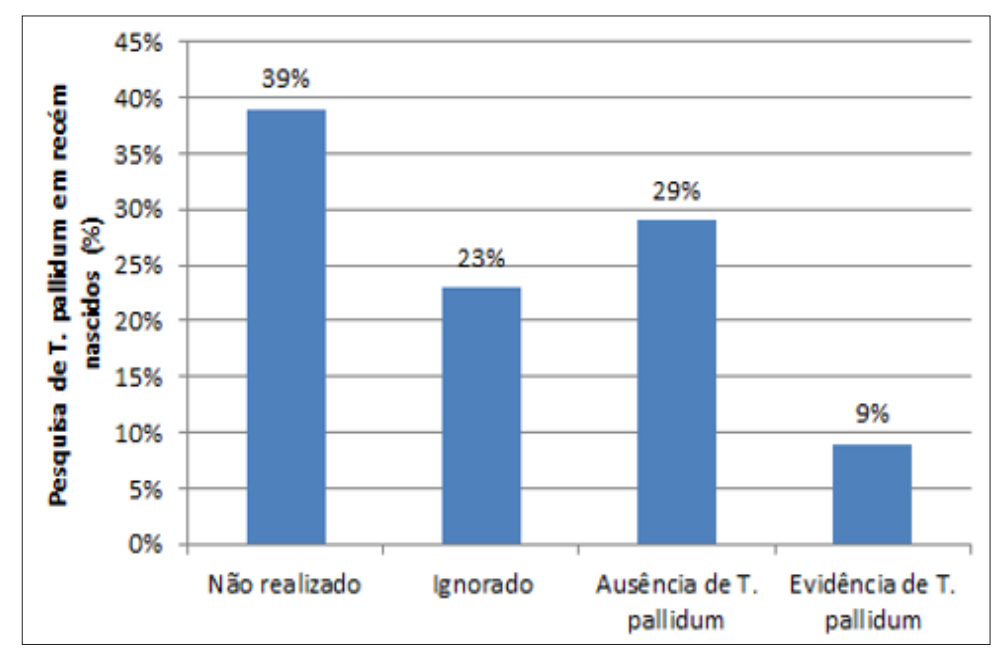

Figura 2. Percentual da realização do exame de evidência do Treponema pallidum em recém-nascidos com síflis congênita no município de Maringá-PR, entre os anos de 2013 a 2016 
Conforme preconizado pelo Ministério da Saú$\mathrm{de}^{31}$, a utilização de testes sorológicos permanece como sendo a principal forma de se estabelecer o diagnóstico da sífilis congênita, além de sua associação com critérios epidemiológicos e clínicos. Neste estudo foram avaliados em conjunto os testes reagentes de VDRL e FTA-Abs no momento do parto/curetagem com positividade de $76,1 \%$ e $52,2 \%$, respectivamente, o VDRL realizado do soro do bebê (50,7\% de positividade) e o FTA-Abs após 18 meses de nascimento $(0,75 \%$ de positividade).

Em 87\% (117/134) dos casos notificados de sífilis congênita, as mães tiveram o diagnóstico da doença durante a gestação, 9\% (12/134) foram após o parto, 1\% (2/134) não realizou o acompanhamento pré-natal e $2 \%$ (3/134) tiveram essa informação ignorada na ficha de notificação. O esquema de tratamento durante a gestação foi realizado adequadamente em 51,4\% (69/134) das mães, enquanto que 35\% (47/134) receberam tratamento inadequado, 9\% (12/134) não receberam nenhum tipo de tratamento e 4,5\% (6/134) não apresentaram informações a respeito do tratamento em suas fichas de notificação.

\section{DISCUSSÃO}

Os resultados do presente estudo mostram que a prevalência da sífilis gestacional e congênita na cidade de Maringá-PR apresentou crescimento durante os anos estudados. Esta tendência de aumento se repete no país como um todo ${ }^{32-33}$. No Brasil, esta enfermidade é mais concentrada em homens que fazem sexo com homens, mas é nítida a capacidade de transmissão para mulheres em idade reprodutiva e com probabilidade de engravi$\operatorname{dar}^{34}$.

$\mathrm{Na}$ maioria das notificações das gestantes estudadas, a doença foi classificada como sífilis primária, fase em que se encontra o cancro, primeira manifestação clínica da sífilis, além de notificações de fase terciária. Estes dados contrapõem os encontrados na literatura, sugerindo desconhecimento sobre a doença ou preenchimento equivocado da ficha de notificação.

A infecção se apresenta assintomática na gestação, justificando a necessidade do seu rastreio sorológi$\mathrm{co}^{35}$. Os testes VDRL realizados nas gestantes estudadas apresentaram títulos elevados semelhantes aos encontrados em um grupo de gestantes estudadas na cidade de Belo Horizonte - MG, no período de 2010 a 2013, onde a maioria das gestantes apresentaram títulos superiores a 1/8, e apenas $19 \%$ dos parceiros das gestantes receberam tratamento adequado ${ }^{36}$. O tratamento precário das gestantes e dos respectivos parceiros também foi observado por Donalísio et al ${ }^{37}$ na cidade de Sumaré, Estado de São Paulo, o que contribui para a disseminação da doença por não possuir parceiros fixos, ou até mesmo para infecções recorrentes. A não adesão dos parceiros de gestantes ao tratamento de síflis está relacionada com o nível de escolaridade, desconhecimento da doença, atividades laborais, nível de relacionamento com a gestante e ausência no pré-natal ${ }^{38}$.

O rastreio de sífilis na gestação é uma das atividades mais custo-efetivas em saúde pública ${ }^{39}$. Apesar dos avanços tecnológicos que agilizam o diagnóstico e o tratamento da gestante, o controle da sífilis na gestação continua sendo um desafio para a atenção pré-nata ${ }^{33}$. O pré-natal é a forma mais simples de possibilitar um diagnóstico precoce, sendo possível intervir na doença durante a gestação com a finalidade de evitar a infecção do concepto.

A estimativa de sífilis congênita no Brasil é de 12 mil recém-nascidos portadores da doença a cada ano, não apresentando diferenças significativas quando comparado às regiões Norte, Nordeste, Sudeste, Sul e Centro-oeste $\mathrm{e}^{3}$, portanto a transmissão vertical da doença ainda se mantém constante, tendo como explicação, uma qualidade reduzida na atenção pré-natal ${ }^{13}$.

A prevalência acumulada de sífilis congênita encontrada neste estudo foi alta, correspondendo a mais da metade dos conceptos das gestantes diagnosticadas com sífilis. O resultado foi similar ao encontrado por Nonato et al. ${ }^{36}$, ao estimar uma taxa de transmissão vertical de $33,4 \%$.

Com os resultados obtidos foi possível observar que quase metade das gestantes acometidas pela doença tinha baixo grau de instrução, ou seja, tempo de escolaridade inferior ou igual a oito anos. Dados semelhantes foram evidenciados por estudos similares ${ }^{40,41,42}$. Domingues et al. ${ }^{40}$ verificaram que a prevalência de sífilis entre as gestantes no Brasil é de 1,6\%, e se apresenta 3,2 vezes 
maior em gestantes que possuem baixa escolaridade. A baixa escolaridade materna é um fator importante que pode predispor ao aparecimento de situações potencialmente de risco para a mãe e o recém-nascido, pois está associada ao baixo peso ao nascer, à mortalidade infantil e ao aumento do número de partos ${ }^{43}$.

Um estudo realizado na cidade de Palmas (TO) relatou 171 casos de sífilis congênita com uma relação na faixa etária materna de 20 a 34 anos com maior prevalência da infecção ${ }^{44}$, dados que corroboram com os encontrados neste estudo. A faixa etária da maior prevalência pode estar relacionada ao período considerado reprodutivo da mulher. Desta forma, as mulheres jovens estão mais propensas a adquirir a infecção, pois este é o período em que estão sexualmente ativas ${ }^{42}$.

Foi verificado aumento significativo dos casos de sífilis congênita entre os anos 2013 (8,2\%) para 2016 $(\mathrm{n}=41,8 \%)$. Esta prevalência crescente também foi observada por Silva et $\mathrm{al}^{42}$ entre os anos de 2007 e 2016 e em um estudo de uma década (2000 a 2009), com um significativo aumento das notificações ano a ano ${ }^{45}$.

O diagnóstico da sífilis congênita representa um processo complexo, pois mais da metade das crianças é assintomática ao nascimento e, mesmo naquelas com alguma expressão clínica, os sinais e sintomas costumam ser discretos ou inespecíficos. A maioria dos recém-nascidos vivos deste estudo foi assintomática. Os dados reforçam que a maior parte dos casos de sífilis congênita ocorre em recém-nascidos aparentemente normais, aumentando a importância da definição de caso epidemiológico ${ }^{37}$.

O Ministério da Saúde lançou em 1993 um projeto de eliminação da sífilis congênita, preconizando condutas que devem ser tomadas para a gestante e recém-nascido, como diagnóstico e tratamento para a sífilis, evidência clínica, laboratorial e radiográfica para sífilis no recém-nato ${ }^{46}$. Porém, a transmissão vertical da doença ainda se mantém constante, tendo como explicação uma qualidade reduzida na atenção pré-natal ${ }^{13}$, característica visível no município estudado, indicando uma deficiência na qualidade do pré-natal tanto na realização de exames quanto no tratamento dos casos confirmados da enfermidade.

A deficiência do tratamento medicamentoso dos doentes e da adoção das políticas públicas de prevenção da doença contribui para o aumento de sífilis congênita. Em uma análise feita pela comparação dos prontuários e cartões do pré-natal de gestantes, observou baixas coberturas do pré-natal nas regiões Norte, Nordeste e Centro-oeste, com destaque para a região Sul, onde as mulheres mais acometidas são brancas e realizaram pré-natal nas redes públicas de saúde ${ }^{40}$. Estes resultados da região Sul corroboram com os encontrados no presente estudo.

Entre as limitações do presente estudo nota-se a utilização de dados secundários de notificação. Apesar da sífilis congênita ser uma doença de notificação compulsória, o sistema de notificação da doença ainda é falho havendo subnotificação de casos. Outra limitação foi a existência de grande número de informações insuficientes ou ignoradas, mostrando a necessidade de melhorias.

\section{CONCLUSÃO}

Com os resultados do presente estudo foi possível observar que houve aumento significativo de sífilis gestacional e congênita, o que indica alguns pontos frágeis na assistência pré-natal e saúde da gestante, que necessitam de melhoras. Quanto às notificações da doença, pode se averiguar que existe falta de informações, o que pode ser prejudicial ao sistema de agravos de notificações compulsórias.

Para redução dos casos dessa enfermidade, a primeira medida a ser tomada é o uso de preservativos, uma vez que o método de contracepção oral previne apenas a gravidez e não a transmissão de infecções sexualmente transmissíveis. Outra maneira seria a procura precoce pelo atendimento pré-natal, realização de exames e tratamento adequado. 


\section{REFERÊNCIAS}

1. Cavalcante AES, Silva MAM, Rodrigues ARM, Netto JJM, Moreira AC, Goyanna NF. Diagnóstico e tratamento da sífilis: uma investigação com mulheres assistidas na atenção básica em Sobral, Ceará. DST - J. bras. Doenças Sex. Transm.2012;24(4):239-45.

2. World Health Organizations (WHO). Sexually Transmitted Infections (STIs).Geneva: World Health Organization; 2008

3. Brasil. Ministério da Saúde, Secretaria de Vigilância em Saúde. Boletim Epidemiológico de Síflis. Brasília: Ministério da Saúde; 2016

4. Rompalo A. Preventing sexually transmitted infections: backtobasics.J Clin Invest. 2011;121(12):45803.

5. Carvalho PMRS, Guimarães RA, Moraes PA, Teles AS, Matos MA. Prevalência de sinais e sintomas e conhecimento sobre doenças sexualmente transmissíveis. Acta. Paul. Enferm. 2015;28(1):95-100.

6. Junior WB, Shiratsu R, Pinto V. Abordagem nas doenças sexualmente transmissíveis. An Bras Dermatol. 2009;84(2):151-9.

7. Lafetá KRG, Júnior HM, Silveira MF, Paranaíba LMR. Sífilis materna e congênita, subnotificação e difícil controle. Rev. Bras. Epidemiol. 2016;19(1):63-74.

8. Brasil. Ministério da Saúde, Secretaria de Vigilância em Saúde. Boletim Epidemiológico de Sífilis. Brasília: Ministério da Saúde; 2015.

9. Santos VC, Anjos KF. Síflis: Uma realidade previnível. Sua erradicação, um desafio atual. Rev Saúde e Pesquisa. 2009;2(2):257-63.

10. Damasceno ABA, Monteiro DLM, Rodrigues LB, Barmpas DBS, Cerqueira LRP, Trajano AJB. Sífilis na gravidez. Rev HUPE. 2014;13(3):88-94.

11. Sonda EC, Richter FF, Boschetti G, Casasola MP, Krumel CF, Machado CPH. Sífilis Congênita: uma revisão da literatura. RevEpidemiolControlInfect. 2013;3(1):28-30.

12. Oliveira FL, Silveira LKCB, Nery JAC. As diversas apresentações de sífilis secundária. Relatos de casos. Rev
Bras Clin Med. 2010;10(6):550-3.

13. Leite I, Oliveira JM, Leão MCM, Lopes SF, França AMB. Assistência da enfermagem na sífilis na gravidez: uma revisão integrativa. Cad Graduação Ciências Biológicas e da Saúde. 2016;3(3):165-76.

14. Brasil. Ministério da Saúde, Secretaria de Vigilância em Saúde. Sífilis: Estratégias para diagnóstico no Brasil. Brasília: Ministério da Saúde; 2010.

15. Feitosa JAS, Rocha CHR, Costa FS. Artigo de revisão: Sífilis congênita. Rev Med Saude Brasilia. 2016;5(2):28697.

16. Saraceni V, Guimarães MHFS, Filha MMT, Leal MC. Mortalidade perinatal por sífilis congênita: indicador da qualidade da atenção à mulher e à criança. Cad Saúde Pública. 2005;21(4):1244-50.

17. Magalhães DMS, Kawaguchi IAL, Dias A, Calderon IMP. A sífilis na gestação e sua influência na morbimortalidade materno-infantil. Comum Ciênc Saúde. 2011;22(1):43-54.

18. Tabisz L, Bobato CT, Carvalho MFU, Takimura M, Reda S, Pundek MRZ. Sífilis, uma doença reemergente. Rev Med Res. 2012;14(3):165-72.

19. Komka MR, Lago EG. Síflis congênita: notificação e realidade. Scientia Medica. 2007;17(4):205-11.

20. Rodrigues LSA, Paiva MS, Oliveira JF, Nóbrega SM. Vulnerabilidade de mulheres em união heterossexual estável à infecção pelo HIV/Aids: estudo de representações sociais. Rev Esc Enferm USP. 2012;46(2):34955. 2012.

21. Costa MC, Azulay DR, Dias MFRG, Demarch EB, Périssé ARS, Nery JAC. Doenças sexualmente transmissíveis na gestação: uma síntese de particularidades. An Bras Dermatol. 2010;85(6):767-85.

22. Domingues RMSM,Saraceni V, Hartz ZMA, Leal MC. Síflis congênita: evento sentinela da qualidade da assistência pré-natal.Rev Saúde Pública 2013;47(1):14757.

23. Brasil. Ministério da Saúde, Secretaria de Vigilância em Saúde. Boletim Epidemiológico de Síflis. Brasília: Ministério da Saúde; 2017. 
24. Hebmuller MG, Fiori HH, Lago EG. Gestações subsequentes em mulheres que tiveram sífilis na gestação. Ciênc saúde coletiva. 2015;20(9):2867-78.

25. São Paulo. Governo do Estado de São Paulo. Secretaria de Saúde. Projeto "Linha de cuidado à gestante, parturiente e puérpera no SUS/SP. Pré-natal e puerpério: manual técnico. São Paulo (SP): Governo do Estado; 2017.

26. Brasil. Ministério da Saúde, Secretaria de Vigilância em Saúde. Protocolo para a prevenção de transmissão vertical de HIV e sífilis. Brasília: Ministério da Saúde; 2007.

27. Carvalho IS, Brito RS. Síflis congênita no Rio Grande do Norte: estudo descritivo do período 2007-2010. Epidemiol Serv Saúde. 2014;23(2):287-94.

28. Pires ACS, Oliveira DD, Rocha GMNM. Ocorrência de sífilis congênita e os principais fatores relacionados aos índices de transmissão da doença no Brasil da atualidade. Uningá Review. 2014;19(1):58-64.

29. Campos ALA, Araujo MAL, Melo SP, Gonçalves MLC. Epidemiologia da sífilis gestacional em Fortaleza, Ceará, Brasil: um agravo sem controle. Cad Saúde Pública. 2010;26(9):1747-55.

30. Araujo EC, Costa KSG, Silva RS, Azevedo VNG, Lima FAS. Importância do pré - natal na prevenção da sífilis congênita. Rev Para Med. 2006;20(1):47-51.

31. Brasil. Ministério da Saúde, Secretaria de Vigilância em Saúde. Protocolo clínico e diretrizes terapêuticas para prevenção da transmissão vertical de HIV, Sífilis e Hepatites virais. Brasília: Ministério da Saúde; 2018.

32. Boni SM, Pagliari PB. Incidência de sífilis congênita e sua prevalência em gestantes em um município do noroeste do Paraná. Saúde e Pesquisa. 2016;9(3):51724.

33. Saraceni V, Pereira GFM, Silveira MF, Araujo MAL, Miranda AE. Vigilância epidemiológica da transmissão vertical da sífilis: dados de seis unidades federativas no Brasil. Rev Panam Salud Publica. 2017;41:e44.

34. Fernandes FR, Zanini PB, Rezende GR, Castro LS, Bandeira LM, Puga MA, Tanaka TS, Castro LS, Bertolacci-Rocha LG, Teles SA, Motta-Castro AR. Syphilis infection, sexual practices and bisexual behaviour among men who have sex with men and transgender women: a cross-sectional study. Sex Transm Infect. 2015;91(2):142-9.

35. Goldenberg RL, Culhane JF, Johnson DC. Maternal infection and adverse fetal and neonatal outcomes. Clin Perinatol. 2005;32(3):523-59.

36. Nonato SM, Melo APS, Guimarães MDC. Sífilis na gestação e fatores associados a sífilis congênita em Belo Horizonte - MG, 2010-2013. Epidemiol Serv Saúde. 2015;24(4):681-94.

37. Donalísio MR, Freire JB, Mendes ET. Investigação da sífilis congênita na microrregião de Sumaré, Estado de São Paulo, Brasil - desvelando a fragilidade do cuidado à mulher gestante e ao recém-nascido. Epidemiol Serv Saúde. 2007;16(3):165-73.

38. Figueiredo MSN, Cavalcante EGR, Oliveira CJ, Monteiro MDFV, Quirino GS, Oliveira DR. Percepção de enfermeiros sobre a adesão ao tratamento dos parceiros de gestantes com sífilis. Rev da Rede de Enfermagem do Nordeste. 2015;16(3):345-54.

39. Kamb ML, Newman LM, Riley PL, Mark J, Hawkes SJ, Malik T, Broutet N. A road map for the global elimination of congenital syphilis. Obstet Gynecol Int. 2010; 2010.pii: 312798.

40. Domingues RMSM, Szwarcwald CL, Junior PRBS, Leal MC. Prevalência da sífilis na gestação e testagem pré-natal: estudo nascer no Brasil. Rev Saúde Pública. 2014;48(5):766-74.

41. Lima MG, Santos RFR, Barbosa GJA, Ribeiro GS. Incidência e fatores de risco para síflis congênita em Belo Horizonte, Minas Gerais, 2001-2008. Ciênc Saúde Coletiva. 2013;18(2):499-506.

42. Silva LCVG, Teodoro CJ, Silva JK, Sanros DAS, Olinda RA. Perfil dos casos de sífilis congênita em um município do sul de Mato Grosso. J Health NPEPS. 2017;2(2):380-90.

43. Ximenes IPE, Moura ERF, Freitas GL, Oliveira NC. Incidência e controle da sífilis congênita no Ceará. Rev Rene. 2008;9(3):74-80.

44. Cavalcante PAM, Pereira RBL, Castro JGD. Syphilis in pregnancy and congenital syphilis in Palmas,Tocan- 
tins State, Brazil, 2007-2014. Epidemiol Serv Saude. 2017;26(2):1-10.

45. Costa CC, Freitas LV, Sousa DMN, de Oliveira LL, Chagas ACMA, Lopes MVO, Damasceno AKC. Congenital syphilis in Ceará: epidemiological analysis of one decade. Rev esc enferm USP. 2013;47(1):152-9.

46. Magalhães DMS,Kawaguchi IAL, Dias A,Calderon IMP. Síflis materna e congênita: ainda um desafio. Cad. Saúde pública. 2013;29(6):1109-20.

Recebido em: 30/05/2018

Aceito em: 08/08/2018 